produced, and the osmotic pressure will rise. Hence liquid will be absorbed from the tube, and the column of air will extend more deeply into the tissues. Moreover, this change will take place first in those regions where the need for oxygen is greatest.

This theory is based chiefly upon experiments with the larva of the mosquito, a detailed account of which will be published shortly. Briefly, it has been found : (i) That in the resting condition the terminal portions of the tracheal tubes are filled with liquid; (ii) that during asphyxiation this liquid is absorbed, and the column of air extends rapidly towards the actively contracting muscles - more slowly and much later towards inactive tissues (for example, the rectal gills) ; (iii) that on readmission of air the level of liquid slowly rises to its original level; (iv) that during asphyxiation an excess of lactic acid is present in the tissue fluids; (v) that hypertonic solutions of sodium chloride and of sodium lactate introduced into the living larva cause a similar extension of air down the tracheal tubes; (vi) that hypotonic fluids (distilled water) are without effect, or cause a slight rise of the liquid in the tracheæ.

It is clear that the mechanism described will serve as a "fine adjustment' for tracheal respiration in insects, just as changes in the capillary bed serve as a 'fine adjustment' for the internal respiration of vertebrates.

London School of Hygiene and

Tropical Medicine, Dec. 5.

\section{Chemical Biogenesis and the Development of Secretion Cells.}

Dr. Lemmann's interesting letter (Nature, Dec. 21 , p. 946) emphasises once more the gains which are bound to accrue from an increasing correlation between organic chemistry, biochemistry, and certain branches of purely biological science. As one who was privileged to obtain a first-hand acquaintance with the classical phyto-chemical researches of R. T. Baker and H. G. Smith on the Australian flora, I am of opinion that such correlation is often realised most effectively by active collaboration between investigators in the related fields. Thus, it may be justly asserted that collaboration between the bistologist and the organic chemist has now become desirable in attacking the engrossing problem of chemical biogenesis (that is, chemical origin in vivo in the terpene series. Nevertheless, one would hesitate to endorse Dr. Leemann's sweeping dictum that the study of such problems "should only [my italies] be done in close connexion with cytology and cell development ". The purely organic chemical aspect of the subject must not be dismissed too lightly. At the present day, after researches extending over half a century, the study in vitro even of such familiar and fundamentally important substances as menthol and menthone is regrettably incomplete. Moreover, of how many essential oils can it be claimed that our formal chemical knowledge is full and adequate?

There is little doubt that a knowledge of the precise cytological origin of the terpenes would be a valuable aid in deciding whether they are derived from carbohydrates (ef. Stewart, "Recent Advances in Organic Chemistry", 1927, vol. 2, pp. 240 and 266), or from protoplasm via amino-acids. Dr. Leemann's suggestion that they originate from protoplasm, rather than from the cell wall, appears to accord with the histological occurrence of fixed oils; while from the chemical point of view leucine offers an attractive approach to the fundamental structural unit (isopentane nucleus) of terpene molecules.

JOHN READ.

\section{Hydrolytic Adsorption at Colloid Surfaces.}

THE outstanding work on hydrolytic adsorption has been carried out with purified charcoal (see, for example, Bartell and Miller, J. Am. Chem. Soc., 44, $1866 ; 1922: 45,1106 ; 1923)$, and definite positive results have been obtained. The measure of the hydrolytic effect in the case of the numerous negative hydrophobic (acidoid) sols, such as colloidal mastic, platinum, gold, arsenious sulphide, etc.-all of which are remarkably alike in structure and in reactions - is complicated by the well-established phenomenon of catalytic interchange. For example, in the presence of salts, such as barium chloride, the hydrogen ion at the colloid surface suffers an interchange with the added barium ion, and the corresponding increase in acidity is superimposed upon any change in acidity due to the hydrolytic action at the surface. It has become the general practice to ascribe the whole of the increase in acidity of acidoid sols (on the addition of neutral salts) solely to the cationic replacement, and to neglect any probable hydrolytic effect. That the latter effect is real is evident from the following consideration.

Colloidal platinum has a great affinity for bases, particularly bases of higher valence such as barium hydroxide, and no measurable affinity for the common inorganic acids such as hydrochloric acid (J.C.S. 551 ; $1928: 618,623 ; 1929)$. This action I have ascribed to the acidic nature of the oxidised platinum which constitutes the greater part of the colloidal platinum surface. It follows from obvious chemical principles, that when a salt such as barium chloride is added to a platinum sol, the barium hydroxide must distribute itself between the hydrochloric acid and the 'acid' surface of the colloid, or in the usual terminology, barium hydroxide must be hydrolytically adsorbed by the platinum. The hydrochloric acid is, of course, a very strong acid, but the affinity of the platinum surface for barium hydroxide has also been shown to be very great. Work recently carried out by me, and to be published in due course, shows clearly that in the case of colloidal platinum (1) such a distribution does take place, (2) its effect is not negligible when compared with the cationic interchange, and (3) it plays an important part in coagulation and reversal phenomena. The same conclusions will probably apply, in varying degree, to the other acidoid sols.

Adelaide, South Australia,

\section{S. W. Pennycuick.} Nov. 5 .

\section{The "Encyclopædia Britannica".}

As a contributor to the new edition of the "Encyclopædia Britannica", may I beg the hospitality of the columns of NATURE in order to disclaim responsibility for certain statements that appear in one of the articles over my initials ? In the article "Acanthocephala" the short passage commencing with the words " the larva of Echinorhynchus gigas ...." and ending at ". . . found in the seal " is not mine.

I should like to add that the editor of the " Encyclopædia ", with whom I have been in communication, has expressed his sincere regret at the unfortunate interpolation, which appears to have occurred in some way during the final making-up of the page, and has undertaken to delete it in future printings of the edition.

British Museum (Natural History) H. A. Baylis. Dec. 17. 